\title{
Percepção da Doença e Enfrentamento em Mulheres com Câncer de Mama
}

\author{
Elisa Kern Kern de Castro ${ }^{1}$ \\ Priscila Lawrenz \\ Fernanda Romeiro \\ Natália Britz de Lima \\ Universidade do Vale do Rio dos Sinos \\ Sílvia Abduch Haas \\ Hospital Santa Rita. Brasil
}

\begin{abstract}
RESUMO - O objetivo do estudo foi investigar a relação entre percepção da doença, enfrentamento e variáveis sociodemográficas e clínicas em mulheres com câncer de mama. O delineamento foi transversal, correlacional e comparativo, do qual participaram 157 mulheres (idade média=51,95 anos). Os questionários de dados sociodemográficos e clínicos, de percepção da doença e de enfrentamento foram aplicados individualmente nos dias de consultas médicas. Os resultados indicaram a existência de relação entre percepção da doença e enfrentamento. A percepção de duração cíclica da doença foi preditora de enfrentamento instrumental e distração. Já a representação emocional foi preditora de enfrentamento emocional. Conclui-se que, apesar de a percepção da doença ser preditora de enfrentamento, a magnitude é fraca.
\end{abstract}

Palavras-chave: neoplasias, neoplasias mamárias, paciente de câncer, atitudes frente à doença, percepção da doença, enfrentamento

\section{Illness Perception and Coping in Women with Breast Cancer}

\begin{abstract}
The objective of the study was to investigate the relationship between illness perception and coping in women with breast cancer. Transversal, correlational and comparative design including 157 women with an average age of 51.95 years at the time of application of the instruments. The sociodemographic and clinical questionnaire of illness perception and coping were applied individually during medical consultation in a specialized cancer hospital. Results indicated the existence of a relationship between illness perception and coping. Perception of cyclic duration was predictive of instrumental coping and distraction. Emotional representation was a predictor of emotional confrontation.It was concluded that, despite illness perceptions being a predictor of coping, the magnitude is low.
\end{abstract}

Keywords: neoplasms, breast neoplasms, cancer patients, physical illness (attitudes toward), illness perception, coping behavior

O câncer de mama é o segundo tipo de câncer mais comum entre mulheres no Brasil e no mundo depois do de pele não melanoma. Estima-se que, somente no ano de 2016, sejam diagnosticados 57,960 novos casos de câncer de mama no Brasil (Instituto Nacional do Câncer [INCA], 2016).

O surgimento da doença é multicausal. História reprodutiva, fatores ambientais e genéticos estão relacionados ao aumento de risco da doença. O câncer de mama é relativamente raro antes dos 35 anos e, acima dessa idade, sua incidência cresce progressivamente, especialmente depois dos 50 anos (INCA, 2016).

A multiplicidade de fatores envolvidos no surgimento do câncer de mama dificulta a sua prevenção. Em $90 \%$ dos casos, a doença se manifesta pela presença de nódulos, que podem ser percebidos pela mulher através do autoexame ou de exames de imagem. Outros sinais e sintomas são: pele da mama avermelhada, alterações no mamilo, pequenos nódulos nas axilas ou no pescoço e saída de líquido anormal das mamas (INCA, 2016).

O diagnóstico e o tratamento do câncer de mama podem impactar a vida da mulher devido aos procedimentos médicos

1 Endereço para correspondência: Programa de Pós-Graduação em Psicologia, Universidade do Vale do Rio dos Sinos, Avenida Unisinos, 950, bairro Cristo Rei, São Leopoldo, RS, Brasil. CEP 93022-000. E-mail: elisakc@unisinos.br invasivos, às alterações corporais, às mudanças nos papeis familiares e às interferências da doença na rotina diária (Belcher et al., 2011). As alterações na imagem corporal que a doença acarreta podem levar a mulher a vivenciar problemas na sexualidade e feminilidade, prejudicando sua qualidade de vida (Boquiren et al., 2015; Farthmann et al., 2016).

A forma como pessoas doentes e saudáveis percebem a doença tem sido investigada seguindo o modelo proposto por Leventhal, Nerenz e Steele (1984). O Modelo do Senso Comum (MSC), também chamado de Modelo da Autorregulação do Comportamento em Saúde, propõe que estímulos de ameaça contra a saúde (p. ex., diagnóstico de câncer de mama) geram a ativação e o desenvolvimento de representações sobre determinada doença a partir de crenças individuais (Leventhal, Brissette, \& Leventhal, 2003; Leventhal et al., 1984). As crenças são estruturadas segundo experiências anteriores e têm influência sobre os comportamentos e o enfrentamento da situação (Figueiras, Machado, \& Alves, 2002).

De acordo com o MSC, o ser humano é concebido como um ente ativo que desenvolve teorias próprias acerca do seu estado de saúde, esforçando-se para dar sentido às experiências corporais. Esse modelo propõe dois processos paralelos, relativamente independentes, mas em contínua interação: o primeiro faz referência à representação mental 
dos elementos objetivos da enfermidade (percepção cognitiva) e o desenvolvimento de ações que visam manejar a ameaça (enfrentamento); o segundo implica a representação mental dos elementos emocionais (percepção emocional) que surgem a partir da enfermidade e a valoração de determinadas estratégias de enfrentamento para lidar com as emoções (Quiles-Marcos, Terol- Cantero, \& Marzo-Campos, 2009).

Portanto, a partir da recepção da informação (interna ou externa ao indivíduo), são produzidos dois processos paralelos de percepção, aos quais se segue uma fase de enfrentamento e outra de evolução da enfermidade, processo em que existe uma retroalimentação contínua que permite a autorregulação do comportamento (Santos, Pais-Ribeiro, \& Lopes, 2007). Nesse sentido, a percepção sobre a doença refere-se à maneira como o indivíduo pensa sobre o seu problema de saúde e, consequentemente, como se comporta diante dele (Leventhal et al., 1984; Leventhal et al., 2003).

Inicialmente, foram identificadas cinco dimensões da percepção da doença (Leventhal et al., 1984): (a) identidade (nome que o indivíduo dá para a sua doença e o conhecimento que possui sobre o seu nível de ameaça e seus sintomas); (b) duração; (c) causas; (d) consequências; e (e) controle/cura (grau de cura e tratamento da doença). Posteriormente, MossMoris et al. (2002) dividiram a dimensão controle/cura em controle pessoal (o quanto a pessoa crê que pode controlar ou curar a doença) e controle do tratamento (crença sobre a eficácia do tratamento no controle ou cura da doença). Além disso, foram incluídas no modelo as dimensões coerência da doença (percepção de quanto o individuo compreende a doença) e representação emocional (emoções negativas relacionadas à doença).

Há uma relação explícita entre percepção e enfrentamento da doença (Scarloo \& Kaptein, 2013). Os indivíduos utilizam uma variedade de estratégias de enfrentamento para lidar com o problema de saúde de acordo com a sua personalidade e a partir do que acreditam que seja eficaz. De acordo com Lazarus e Folkman (1984), enfrentamento é compreendido como o conjunto de estratégias cognitivas e comportamentais utilizadas em resposta a eventos estressantes. Consiste em um processo de mudanças voltadas a lidar com demandas internas e/ou externas que são avaliadas de acordo com os recursos de cada indivíduo. As estratégias de enfrentamento adaptativas estão associadas ao uso de formas variadas e múltiplas, ajustadas ao estresse da situação.

Segundo Endler, Kocovski e Macrodimitris (2001), frente a uma doença, os indivíduos utilizam quatro grandes estratégias de enfrentamento: (a) distração: pensar ou comprometer-se em atividades que não sejam relacionadas ao problema de saúde; (b) paliativo: estratégias calmantes que objetivam aliviar o aborrecimento com o problema de saúde; (c) instrumental: envolve estratégias como procurar mais informações sobre a doença ou buscar conselhos médicos; (d) preocupação emocional: envolve o foco nas consequências emocionais da doença.

A relação entre percepção da doença e enfrentamento em mulheres com câncer de mama foi investigada por Rozema, Völlnik e Lechner (2009). De acordo com esse estudo, a percepção de controle pessoal e a atribuição de causas psicológicas à doença foram preditoras de enfrentamento focado na resolução de problemas. Já McCorry et al.
(2013), em estudo longitudinal (seis meses) que avaliou percepção da doença, coping e distress psicológico, não encontraram evidências de que mudanças nessas percepções e no enfrentamento estivessem associadas com sintomas psicológicos.

Diante disso, este estudo teve como objetivo investigar a relação entre percepção da doença, enfrentamento e variáveis sociodemográficas e clínicas em mulheres com diagnóstico de câncer de mama. Buscou-se, também, verificar o possível papel preditor da percepção da doença para o uso de estratégias de enfrentamento, controlando as variáveis sociodemográficas e clínicas.

\section{Método}

\section{Delineamento}

Transversal e correlacional.

\section{Amostra}

Participaram deste estudo 157 mulheres diagnosticadas com câncer de mama. A amostra foi selecionada de forma consecutiva entre mulheres adultas (entre 18 e 65 anos) que estavam em tratamento oncológico em um hospital especializado localizado numa capital do sul do Brasil.

No momento do diagnóstico, a média de idade das participantes era de 49,33 anos $(D P=8,73)$ e, no momento da entrevista, de 51,95 anos $(D P=8,03)$. A maioria delas era casada ou vivia com o companheiro $(64,3 \%)$ e tinha filhos $(84,7 \%)$ com os quais morava $(56,1 \%)$. A maior parte das participantes não trabalhava $(68,2 \%)$ e $33,1 \%$ não concluíram o ensino fundamental. Em relação aos dados clínicos, o tipo de câncer mais incidente foi o carcinoma ductal infiltrante (66,9\%), seguido pelo carcinoma lobular infiltrante $(10,8 \%)$, carcinoma ductal in situ $(10,8 \%)$, carcinoma lobular in situ $(0,6 \%)$ e carcinoma medular $(0,6 \%)$. A maior parte das participantes não apresentava recidiva $(96,2 \%)$ ou metástase ( $74,5 \%)$, sendo que, em $15,9 \%$ dos casos, o câncer de mama estava em estadiamento I; em 29,3\%, em estadiamento II; em $17,2 \%$, em estadiamento III; e, em $22,3 \%$, em estadiamento $\mathrm{IV}^{1}$. A quimioterapia $(65 \%)$ e a radioterapia $(70,1 \%)$ foram os tratamentos mais frequentes. No caso da cirurgia, $84,1 \%$ das pacientes realizaram mastectomia e somente $10,2 \%$ já haviam realizado cirurgia reconstrutiva da mama. Na Tabela 1 são apresentadas as características sociodemográficas e clínicas das participantes.

\section{Instrumentos}

Questionário de dados sociodemográficos e clínicos. Incluiu dados sobre idade, estado civil, filhos, atividade laboral, tipo de tratamento realizado e estadiamento da

1 Em 15,3\% dos casos, não foram encontrados os dados referentes ao estadiamento nos prontuários 
doença. Os dados foram obtidos junto aos prontuários e por meio do autorrelato das participantes.

Revised Illness Perception Questionnaire (IPQ-R; Moss-Morris et al., 2002). Avalia a percepção da doença. Possui uma versão adaptada para o português por Figueiras e Alves (2007) e uma validação para pacientes oncológicos realizada por Santos, Pais-Ribeiro e Lopes (2003). É constituído por três dimensões que são divididas em subescalas: (a) identidade da doença (lista de sintomas que a pessoa deve atribuir ou não à doença); (b) duração cíclica, duração aguda/crônica, consequências, controle pessoal, controle do tratamento, coerência da doença e representação emocional (escala Likert de um a cinco pontos); (c) causas da doença. Ao final, o participante deve elencar as principais causas que acredita terem contribuído para o surgimento da doença. Quanto maior a pontuação, mais percepções negativas o indivíduo tem da sua doença. Os alphas encontrados foram de 0,70 (duração cíclica), 0,73 (duração agudo/crônico), 0,65 (consequências), 0,30 (controle pessoal), 0,54 (controle do tratamento), 0,75 (coerência da doença) e 0,77 (representação emocional). Optou-se por analisar neste estudo apenas as dimensões com alphas superiores a 0,60, levando à exclusão das dimensões controle pessoal e controle do tratamento.

Tabela 1 Dados sociodemográficos e clínicos das participantes

\begin{tabular}{|c|c|}
\hline & $\mathbf{N}(\%)$ \\
\hline \multicolumn{2}{|l|}{ Estado Civil } \\
\hline Casada/Vive com companheiro & $101(64,3)$ \\
\hline Separada & $22(14)$ \\
\hline Viúva & $18(11,5)$ \\
\hline Solteira & $16(10,2)$ \\
\hline \multicolumn{2}{|l|}{ Escolaridade } \\
\hline Até ensino fundamental completo & $75(47,8)$ \\
\hline Até ensino médio completo & $46(29,3)$ \\
\hline Até ensino superior completo & $36(22,9)$ \\
\hline \multicolumn{2}{|l|}{ Trabalha } \\
\hline Sim & $50(31,8)$ \\
\hline \multicolumn{2}{|l|}{ Não } \\
\hline \multicolumn{2}{|l|}{ Filhos } \\
\hline Sim & $106(67,5)$ \\
\hline \multicolumn{2}{|l|}{ Não } \\
\hline \multicolumn{2}{|l|}{ Tratamento } \\
\hline Quimioterapia & $102(65)$ \\
\hline Radioterapia & $110(70)$ \\
\hline Matectomia & $132(84,1)$ \\
\hline Hormonioterapia & $38(24,2)$ \\
\hline Cirurgia reconstrutiva da mama & $16(10,2)$ \\
\hline \multicolumn{2}{|l|}{ Presença de recidiva } \\
\hline Sim & $6(3,8)$ \\
\hline \multicolumn{2}{|l|}{ Não } \\
\hline \multicolumn{2}{|l|}{ Presença de metástase } \\
\hline Sim & $35(22,3)$ \\
\hline Não & \\
\hline
\end{tabular}

Coping with Health Injuries and Problems (CHIP; Endler et al., 1998). Avalia as estratégias de enfrentamento empregadas por indivíduos frente a determinado problema de saúde. O instrumento, originalmente em inglês, não está validado no Brasil e foi construída uma versão para o português através de back-translation. Possui 32 itens divididos em quatro dimensões: estratégias de enfrentamento distrativas, paliativas, instrumentais e preocupações emocionais. As respostas são dadas a partir de uma escala Likert, de um (nada) a cinco (completamente). Maiores pontuações na escala indicam maior uso do enfrentamento em questão. No presente estudo, os alphas encontrados foram de 0,91 (enfrentamento instrumental), 0,84 (enfrentamento distração), 0,74 (enfrentamento preocupação emocional) e 0,44 (enfrentamento paliativo). Em função do baixo valor do alpha do enfrentamento paliativo, decidiu-se excluir essa subescala das análises.

\section{Procedimentos Éticos e de Coleta dos Dados}

O projeto foi aprovado por Comitê de Ética. A coleta de dados foi realizada no período de seis meses em um hospital especializado no tratamento de pacientes oncológicos. As participantes foram selecionadas por meio da leitura dos prontuários e a aplicação dos instrumentos foi realizada em uma sala do ambulatório do setor de oncologia. No contato com a paciente, foram explicados os objetivos da pesquisa e solicitada sua participação voluntária. A fim de padronizar a aplicação dos instrumentos, as pesquisadoras leram os itens para todas as participantes em função da dificuldade de leitura que algumas delas apresentavam.

\section{Análise dos Dados}

Os dados foram analisados através do pacote estatístico SPSS 20.0. Foram realizadas análises descritivas dos resultados em geral (médias, valores máximos e mínimos e desvio padrão). O Teste $t$ de Student para amostras independentes foi utilizado para comparar as percepções e o enfrentamento entre mulheres com e sem companheiro, mulheres que realizaram ou não a quimioterapia, e de acordo com o estadiamento da doença (I e II versus III e IV). A análise de correlação de Pearson relacionou as dimensões da percepção da doença e as estratégias de enfrentamento. As análises de regressão múltipla seguiram o método de passos sucessivos (stepwise) para analisar o poder preditivo da percepção sobre a doença nas estratégias de enfrentamento, controladas as variáveis sociodemográficas e clínicas.

As respostas abertas das questões sobre as causas do câncer foram analisadas conforme as categorias do estudo de Figueiras e Alves (2007): (a) estado emocional/ comportamento; (b) hereditariedade/alterações nas defesas do organismo; (c) causas externas ao indivíduo; (d) problemas conjugais/familiares; (e) maus hábitos de saúde/ má alimentação; (f) destino/má sorte; $(\mathrm{g})$ pouca assistência médica; (h) excesso de trabalho; (i) não sabe; (j) outros. 


\section{Resultados}

Na Tabela 2, são apresentadas as médias, o desviopadrão e os valores mínimos e máximos dos resultados das subescalas do IPQ-R e CHIP.

Com relação à dimensão identidade da doença, os sintomas que as pacientes indicaram estar relacionados ao câncer de mama, em ordem decrescente, foram: dores $(60,5 \%)$, cansaço $(63,7 \%)$, perda de forças $(60,5 \%)$, rigidez nas articulações $(51 \%)$, náusea $(51 \%)$, dificuldades para dormir $(47,8 \%)$, indisposição do estômago $(46,5 \%)$, perda de peso $(42,3 \%)$, tonturas $(34,4 \%)$, dores de cabeça $(30,6 \%)$ e falta de ar $(24,4 \%)$.

Com relação às respostas sobre as possíveis causas da doença (apresentadas na Tabela 3), o estado emocional/ comportamento $(41,1 \%)$ foi a resposta mais frequente entre as participantes, seguida por hereditariedade/alterações das defesas do organismo $(24,8 \%)$ e causas externas ao indivíduo (7\%). Já nas segundas respostas espontâneas, $34,4 \%$ atribuíram ao estado emocional/comportamento a possível causa para o surgimento da doença e $26,8 \%$ não souberem responder. $\mathrm{O}$ mesmo ocorreu na terceira resposta espontânea, com mais da metade das participantes $(52,2 \%)$ sem saber mencionar uma causa para o câncer de mama.

Realizou-se o Teste $t$ de Student a fim de comparar os resultados da percepção sobre o câncer de mama e do enfrentamento em relação às variáveis sociodemográficas

Tabela 2. Dados descritivos (médias, desvio padrão, mínimo e máximo) das subescalas da percepção da doença (IPQ-R) e enfrentamento (CHIP)

\begin{tabular}{lcccc}
\hline & Média & DP & Min & Max \\
\hline IPQ-R duração agudo/crônica & 14,66 & 5,290 & 6 & 30 \\
IPQ-R duração cíclica & 13,02 & 3,980 & 4 & 20 \\
IPQ-R consequências & 19,70 & 5,161 & 6 & 30 \\
IPQ-R coerência da doença & 16,45 & 4,507 & 6 & 25 \\
IPQ-R representação emocional & 17,70 & 5,745 & 6 & 30 \\
IPQ-R identidade & 11,99 & 6,027 & 0 & 27 \\
CHIP instrumental & 19,15 & 11,174 & 8 & 40 \\
CHIP distração & 19,87 & 9,442 & 8 & 40 \\
CHIP preocupação emocional & 24,12 & 8,316 & 8 & 40 \\
\hline
\end{tabular}

e clínicas. Verificou-se que mulheres casadas/que viviam com o companheiro percebiam mais as consequências negativas da doença que as solteiras, viúvas ou separadas ( $t=$ $-2,434, p<0,05)$. Por outro lado, mulheres com diagnósticos mais avançados (estadiamentos III e IV no momento do diagnóstico do câncer) percebiam a doença com mais consequências negativas $(t=-2,850, p<0,01)$ e apresentavam mais percepções emocionais negativas $(t=-2,227, p<0,05)$ que as mulheres que, no diagnóstico, tinham a doença em estágios iniciais (I e II). Além disso, pacientes que passaram pelo tratamento da quimioterapia percebiam a doença com duração mais cíclica que as demais $(t=-2,838 ; p<0,01)$.

Com respeito às correlações entre as dimensões da percepção da doença e o enfrentamento, constatou-se que o enfrentamento instrumental correlacionou-se negativamente com a duração cíclica da doença $(r=-0,171 ; p<0,05)$ e com as consequências da doença $(r=-0,170 ; p<0,05)$, o que demonstra que, quanto mais negativa a percepção de duração cíclica e das consequências, menos estratégias de enfrentamento instrumental ou focadas no problema eram utilizadas. $\mathrm{O}$ enfrentamento distração correlacionou-se negativamente com as subescalas duração cíclica da doença $(r=-0,221 ; p<0,01)$ e consequências $(r=-0,162 ; p<0,05)$, ou seja, quanto mais negativa a percepção da paciente a respeito da duração cíclica e das consequências da doença, menor o uso da distração como forma de lidar com a situação. Já a subescala representação emocional correlacionou-se negativamente com o enfrentamento emocional, isto é, quanto mais negativa a representação emocional a respeito da doença, menos empregadas eram as estratégias de enfrentamento emocional $(r=-0,253 ; p<0,01)$. Observaramse, ainda, correlações significativas negativas entre a idade da paciente e as subescalas consequências da doença $(r=-0,185$; $p<0,05)$ e representação emocional $(r=-0,199 ; p<0,05)$. Quanto maior a idade das pacientes, menos negativas eram as suas percepções a respeito dos estados emocionais e das consequências da doença.

Por fim, as análises preditivas de regressão linear demonstraram que a subescala duração cíclica da doença foi a única preditora do uso de enfrentamento instrumental $\left(\beta=-0,171 ; p<0,05 ; R^{2}=0,029 ; p<0,05\right)$ e do enfrentamento distração $\left(\beta=-0,221 ; p<0,01 ; R^{2}=0,049 ; p<0,05\right)$, isto, é, a percepção de que a doença vai e volta prediz tanto o maior

Tabela 3. Fatores considerados pelas pacientes como causadores do câncer de mama, por ordem de resposta

\begin{tabular}{lccc}
\hline \multicolumn{1}{c}{ Causas do câncer } & $\mathbf{1}^{\mathbf{a}}$ Resposta N (\%) & $\mathbf{2}^{\mathbf{a}}$ Resposta N (\%) & $\mathbf{3}^{\mathbf{a}}$ Resposta N (\%) \\
\hline Estado emocional/comportamento & $65(41,4 \%)$ & $54(34,4 \%)$ & $31(19,7 \%)$ \\
Hereditariedade/alterações da defesa do organismo & $39(24,8 \%)$ & $16(10,2 \%)$ & $9(5,7 \%)$ \\
Causas externas ao indivíduo & $11(7 \%)$ & $7(4,5 \%)$ & $1(0,6 \%)$ \\
Problemas conjugais/familiares & $9(5,7 \%)$ & $3(1,9 \%)$ & $3(1,9 \%)$ \\
Não sabe & $8(5,1 \%)$ & $42(26,8 \%)$ & $82(52,2 \%)$ \\
Maus hábitos de saúde/má alimentação & $6(3,8 \%)$ & $14(8,9 \%)$ & $14(8,9 \%)$ \\
Destino/má sorte & $5(3,2 \%)$ & $5(3,2 \%)$ & $1(0,6 \%)$ \\
Pouca assistência médica & $5(3,2 \%)$ & $6(3,8 \%)$ & $2(1,3 \%)$ \\
Outros & $5(3,2 \%)$ & $6(3,8 \%)$ & $5(3,2 \%)$ \\
Excesso de trabalho & $4(2,5 \%)$ & $4(2,5 \%)$ & $9(5,7 \%)$ \\
Total & $157(100 \%)$ & $157(100 \%)$ & $157(100 \%)$ \\
\hline
\end{tabular}


uso de enfrentamento instrumental quanto de distração. Já a subescala representação emocional foi a única preditora do enfrentamento emocional $\left(\beta=0,253 ; p<0,01 ; R^{2}=0,064\right.$; $p<0,01$ ), indicando que pacientes que percebem a sua doença com efeitos emocionais negativos tendem a usar mais o enfrentamento focado na emoção. Apesar desses resultados serem significativos, a magnitude da predição é baixa.

\section{Discussão}

Este estudo investigou a relação entre percepção da doença, enfrentamento e variáveis sociodemográficas e clínicas em mulheres com diagnóstico de câncer de mama, além de verificar o possível papel preditor da percepção da doença para o uso de estratégias de enfrentamento, controlando as variáveis sociodemográficas e clínicas. Os resultados apontaram para a relação entre percepção da doença e enfrentamento. Porém, apenas duas subescalas da percepção (duração cíclica e representação emocional) foram preditoras de enfrentamento, e essa predição foi de baixa magnitude.

A ausência de correlações significativas entre as variáveis da doença e do tratamento e a percepção sobre a doença e o enfrentamento demonstra que as percepções subjetivas das pacientes parecem ter alguma independência em relação aos dados objetivos do seu estado de saúde e às características do tratamento. Essa constatação reforça a ideia de que a percepção sobre a doença pode ter mais peso no momento de enfrentar a doença do que o dado de realidade da enfermidade. Além disso, reafirma uma das ideias centrais do MSC, de que a subjetividade do indivíduo é o motor da autorregulação do seu comportamento em saúde, superando dados concretos (Leventhal, Brissette \& Leventhal, 2003).

Constatou-se que o grupo de mulheres casadas ou que vivem com o companheiro percebeu que o câncer de mama gerava mais consequências negativas quando comparado às mulheres solteiras, viúvas ou separadas. Talvez a existência desse marido/companheiro faça com que as mulheres se deparem de uma forma mais intensa com as mudanças e mutilações corporais causadas pela doença e o tratamento, o que interfere no relacionamento e na sexualidade do casal. O diagnóstico e tratamento de uma doença crônica como o câncer é um evento que gera sofrimento tanto para a pessoa acometida pela enfermidade quanto para o seu cônjuge. Essa experiência tende a gerar dificuldades de comunicação entre os cônjuges, mudança de papeis e diminuição da atividade sexual, pois o companheiro passa a desempenhar o papel de cuidador da mulher enferma (Picheti, Castro, \& Falcke, 2014).

Os resultados também demonstraram que, quanto maior a idade, menos negativa era a representação emocional, a percepção de controle pessoal e de consequências da doença. É possível que o impacto de uma doença grave como o câncer seja maior para pessoas jovens devido ao período do ciclo vital em que se encontram.

Com relação à gravidade da doença, as pacientes com estadiamento avançado (III e IV) perceberam que o câncer de mama gerava mais consequências negativas e mais representações emocionais negativas quando comparadas às pacientes em estágios iniciais (I e II). A enfermidade em estágios avançados requer tratamentos diferenciados que podem gerar efeitos adversos importantes, o que faz com que as experiências subjetivas sejam peculiares e levem a percepções mais negativas sobre suas consequências. $\mathrm{O}$ medo da morte nos casos de doença mais avançada é real, pois as chances de cura da doença diminuem conforme o seu estadiamento (INCA, 2016). Nesse sentido, possivelmente a percepção de consequências negativas da doença em mulheres com câncer de mama avançado reflete a consequência morte.

$\mathrm{O}$ fato de as pacientes que passaram pela quimioterapia perceberem o câncer de mama como mais cíclico se comparadas às mulheres que experienciaram outros tipos de tratamento pode estar relacionado às características dos procedimentos quimioterápicos. A quimioterapia é aplicada em ciclos, os quais podem variar de acordo com o fármaco utilizado, o estadiamento e o tipo de câncer. Os efeitos colaterais desagradáveis são, também, cíclicos (p. ex., enjôos e fraqueza; INCA, 2016). Portanto, é possível que as mulheres que realizam quimioterapia percebam os efeitos colaterais do tratamento como cíclicos, confundindo-os com os sintomas da própria doença.

No que diz respeito às causas da doença atribuídas pelas pacientes, é interessante notar que o estado emocional/ comportamento foi o mais lembrado nas três ordens de respostas. Esse dado evidencia uma dificuldade das mulheres em compreenderem a doença, buscando explicações sobre suas causas que nem sempre condizem com a realidade. Recente estudo que investigou as crenças sobre as causas do câncer de mama evidenciou que a atribuição do câncer a fatores psicológicos é bastante frequente (Peuker, Armiliato, Vital, \& Castro, 2016). Essa percepção distorcida pode gerar sofrimento para as mulheres, uma vez que elas podem se culpabilizar pela sua doença.

Em resumo, os principais resultados deste estudo apontam para a relação entre enfrentamento e percepção sobre a doença, em especial no que se refere às subescalas duração cíclica e representação emocional, embora a magnitude dessa relação seja fraca. A avaliação das percepções e das formas de enfrentamento desse grupo de pacientes torna-se cada vez mais relevante porque fornece informações para as equipes acerca do que pensam as mulheres sobre o seu estado de saúde. Assim, poderão ser elaboradas estratégias de intervenção mais eficazes, que auxiliarão no atendimento de mulheres que passam pela experiência do câncer de mama. Além disso, os resultados evidenciam que os profissionais da saúde devem estar atentos não apenas aos aspectos físicos, mas, também, às percepções subjetivas das pacientes, que podem interferir nas suas condutas de enfrentamento.

Este estudo apresenta algumas limitações que impedem a generalização de seus achados. A amostra foi selecionada de forma consecutiva e, portanto, não é representativa. Além disso, foram usados instrumentos que ainda não foram validados para a população brasileira, embora já tenham sido usados em outro estudos com pacientes com câncer nesse contexto (Peuker et. al., 2016; Castro, Peuker, Lawrenz, \& Figueiras, 2015). Contudo, por se tratar de um tema ainda pouco explorado no Brasil, esses resultados podem ser vistos como um primeiro passo para conhecer a relação entre 
percepções sobre a doença e enfrentamento em situação de câncer de mama nesse contexto.

\section{Referências}

Belcher, A. J., Laurenceau, J., Graber, E. C., Cohen, L. H., Dasch, K. B., \& Siegel, S. D. (2011). Daily support in couples coping with early stage breast cancer: Maintaining intimacy during adversity. Health Psychology, 6, 665-673. doi: 10.1037/ a0024705.

Boquiren, V., Esplen, M. J., Wong, J., Toner, B., Warner, E., \& Malik, N. (2015). Sexual disturbance in breast cancer survivors experience body image disturbance. Psycho-Oncology, 25, 66-76. doi: 10.1002/pon.3819

Castro, E. K., Peuker, A. C., Lawrenz, P. \& Figueiras, M. J. (2015). Illness Perception, Knowledge and Self-Care about Cervical Cancer. Psicologia: Reflexão e Crítica, 28, 483-489. doi 10.1590/1678-7153.20

Endler, N. S., Kocovski, N. L., \& Macrodimitris, S. D. (2001). Coping, efficacy, and perceived control in acute vs chronic illnesses. Personality and Individual Differences, 30, 617-625. doi 10.1016/S0191-8869(00)00060-X.

Endler, N. S., Parker, J. D., \& Summerfeldt, L. J. (1998). Coping with health problems: Developing a reliable and valid multidimensional measure. Psychological Assessment, 10, 195-205. doi: 10.1037/1040-3590.10.3.195.

Farthmann, J., Hanjalic-Beck, A., Veit, J., Rautenberg, B., Stickeler, E., Erbes, T., Földi, M., \& Hasenburg, A. (2016). The impact of chemotherapy for breast cancer on sexual function and health related quality of life. Supportive Care in Cancer, 24, 2603-2609. Doi 10.1007/s00520-015-3073-2. .

Figueiras, M. J., \& Alves, N. C. (2007). Lay perceptions of serious illnesses: An adapted version of the Revised Illness Perception Questionnaire (IPQ-R) for healthy people. Psychology and Health, 22, 143-158.

Figueiras, M. J., Machado, V.A., \& Alves, N. C. (2002). Os modelos do senso-comum das cefaleias crónicas nos casais: Relação com o ajustamento marital. Análise Psicológica, 20(1), 77-90. doi: 10.14417/ap.285

Instituto Nacional do Câncer. (2016). Tipos de câncer. Retrieved from http://www2.inca.gov.br/wps/wcm/connect/tiposdecancer/site/ home/mama
Lazarus, R., \& Folkman, S. (1984). Stress, appraisal, and coping. New York: Springer Publishing Company.

Leventhal, H., Nerenz, D. L., \& Steele, D. J. (1984). Illness representation and coping with health threats. In A. Baum, S. E. Taylor, \& J. E. Singer (Ed.), Handbook of Psychology and Health: Vol. IV. Social Psychological aspects of health (pp. 219-253). Hillsdale, NJ: Erlbaum.

Leventhal, H., Brissette, I., \& Leventhal, E. A. (2003). The commonsense model of self-regulation of health and illness. In L.D. Cameron \& H. Leventhal (Eds.), The self-regulation of health and illness behaviour (pp. 42-65). London: Routledge.

McCorry, N. K., Dempster, M., Quinn, J., Hogg, A., Newell, J., Moore, M. ... Kirk, S. J. (2013). Illness perception clusters at diagnosis predict psychological distress among women with breast cancer at 6 months post diagnosis. Psycho-Oncology, 22(3), 692-698. doi: 10.1002/pon.3054.

Moss-Morris, R., Weinman, J., Petrie, K. J., Horne, R., Cameron, L. D., \& Buick, D. (2002). The Revised Illness Perception Questionnaire (IPQ-R). Psychology and Health, 17, 1-16.

Peuker, A. C., Armiliato, M. J., Vital, L., \& Castro, E. K. (2016). Causal attribution among women with breast cancer. Psicologia Reflexão e Crítica, 29(4). doi: 10.1186/s41155-016-0007-y

Picheti, J., Castro, E. K., \& Falcke, D. (2014) Silêncios e rearranjos na conjugalidade em situação de câncer em um dos cônjuges. Psicologia em Pesquisa (UFJF), 8, 189-199.

Quiles-Marcos, Y., Terol-Cantero, M. C., \& Marzo-Campos, J. C. (2009). Representación de la Enfermedad, Afrontamiento y Ajuste en los Trastornos Alimentarios. Clinica y Salud, 20(2), 159-175.

Rozema, H., Völlink, T., \& Lechner, L. (2009). The role of illness representations in coping and health of patients treated for breast cancer. Psycho-Oncology, 18, 849-857. doi: 10.1002/ pon. 1488 .

Santos, C., Pais-Ribeiro, J., \& Lopes, C. (2003). Adaptação e validação do "Revised Illness Perception Questionnaire" (IPQ-R) em doentes oncológicos. Arquivos de Medicina, 17, 136-147.

Scharloo, M., \& Kaptein, A. (2013). Measurement of illness perception in patients with somatic chronic illness: A review. In K. Petrie \& J. Weinmann (Orgs.), Perception of health and illness: Current research and aplications (pp. 103-152). New York: Routledge. 